\title{
Open magnetic flux and magnetic flux closure during sawtooth events
}

\author{
B. Hubert, ${ }^{1}$ S. E. Milan, ${ }^{2}$ A. Grocott, ${ }^{2}$ S. W. H. Cowley, ${ }^{2}$ and J. C. Gérard ${ }^{1}$ \\ Received 17 October 2008; accepted 29 October 2008; published 3 December 2008.
}

[1] We use IMAGE-FUV observations of the polar aurora and measurements of the ionospheric convection from the SuperDARN radar network to study several sawtooth events previously reported in the literature. We estimate the amount of open magnetic flux in the Earth magnetosphere during a significant part of these sawtooth intervals as well as the magnetic flux opening and closure rates, that is, the dayside and nightside reconnection rates. We find that during the sawtooth intervals the magnetosphere is highly loaded with open flux as a result of the strongly southward IMF carried by the solar wind during these intervals. The magnetosphere tries to relax to a less loaded configuration through a sequence of substorm expansions. However, these substorms do not necessarily evolve to their end before reintensification of nightside reconnection occurs in response to continued loading of the magnetosphere on the dayside. Citation: Hubert, B., S. E. Milan, A. Grocott, S. W. H. Cowley, and J. C. Gérard (2008), Open magnetic flux and magnetic flux closure during sawtooth events, Geophys. Res. Lett., 35, L23301, doi:10.1029/2008GL036374.

\section{Introduction}

[2] A sawtooth event is a prolonged interval during which quasiperiodic large intensifications of the particle fluxes occur at geosynchronous altitude [Belian et al., 1995; Henderson et al., 2006]. Sawtooth events occur generally during storms, when the ring current is enhanced and the IMF is continuously southward (on average) for prolonged intervals of time. The shape of the proton flux vs. time curve during these intervals exhibits quasi-periodic rapid increases followed by slow decreases, resembling the teeth of a saw blade [Henderson et al., 2006]. These events are similar to a sequence of quasi-periodic substorms, although a shock-induced triggering has sometimes been observed [Henderson et al., 2006, and references therein]. Substorms evolve on timescales of about three hours [Akasofu, 1964; McPherron, 1970], and consist essentially in a cycle of open flux production on the dayside and transport to the nightside where it is eventually closed. This process is also primarily responsible for convection in the Earth's magnetosphereionosphere system [Dungey, 1961; Cowley and Lockwood, 1992].

[3] We have developed a method that combines ground based measurements of the ionospheric convection from the Super Dual Aurora Radar Network (SuperDARN) and images of the proton aurora from the Spectrographic Imager

\footnotetext{
${ }^{1}$ Laboratory for Planetary and Atmospheric Physics, University of Liège, Liège, Belgium.

${ }^{2}$ Department of Physics and Astronomy, University of Leicester, Leicester, UK.
}

Copyright 2008 by the American Geophysical Union. 0094-8276/08/2008GL036374\$05.00 at $121.8 \mathrm{~nm}$ (SI12) instrument of the IMAGE-FUV experiment [Mende et al., 2000a, 2000b] to estimate the amount of open magnetic flux in the magnetosphere as well as the rate of production of open magnetic flux on the dayside and of flux closure in the magnetotail [Hubert et al., 2006a]. According to Faraday's law, the rate of change of the open flux can be expressed as a voltage, so that the reconnection rates are given as opening and closure voltages. The SI12 images of the proton aurora are used to estimate the location and motion of the open/closed boundary [Hubert et al., 2006a]. The SuperDARN data are used to obtain the ionospheric electric field [Ruohoniemi and Baker, 1998]. We use this information to compute the electric field in the frame of reference of the moving boundary and finally the opening and closure voltages. In the present study, we apply this method to sawtooth events previously reported in the literature. The WIC and SI13 images of the IMAGE-FUV experiment [Mende et al., 2000a, 2000b] are also used to analyze the development of the auroral activity during the sawtooth event. The WIC and SI13 instruments are sensitive to emissions that are present in the dayglow and in the aurora due to both electron and proton precipitation. The SI12, on the other hand, produces images of the Dopplershifted Lyman- $\alpha$ emission, which is solely produced by the proton aurora. Dayglow contamination of the SI12 images is minor.

\section{Sawtooth Event of $\mathbf{1 8}$ April 2001}

[4] Huang et al. [2004] listed several sawtooth events detected using geosynchronous measurements of particle data. One of them occurred on 18 April 2001 between 00 and 08 UT. During this interval, SI12 images of the proton aurora were collected between 00 and 05 UT, complemented with SuperDARN measurements of the ionospheric convection. This allows us to estimate the magnetic open flux and the opening and closure voltages during a significant fraction of the sawtooth interval, although the SuperDARN coverage was not always good during that time. Figure 1 shows the open flux and reconnection voltages based on these data. The net voltage, which is the sum of the opening and closure voltage, represents the time derivative of the open flux. It depends only on variations of the polar cap size, and does not, therefore, depend on the ionospheric convection, whereas the opening and closure voltages do. At the beginning of the interval, the magnetosphere had accumulated more than $0.8 \mathrm{GWb}$ of open flux (Figure 1a). This is a rather large value, sufficient to power a strong substorm [Milan et al., 2007]. The particle data from the LANL-01A spacecraft suggest that a substorm onset occurs at 0047 UT [Huang et al., 2004]. At that time, a solar wind shock, recorded by the ACE satellite at 0005 UT (Figure 1h), reached the magnetosphere and triggered the process of magnetic flux closure. The shock, associated 

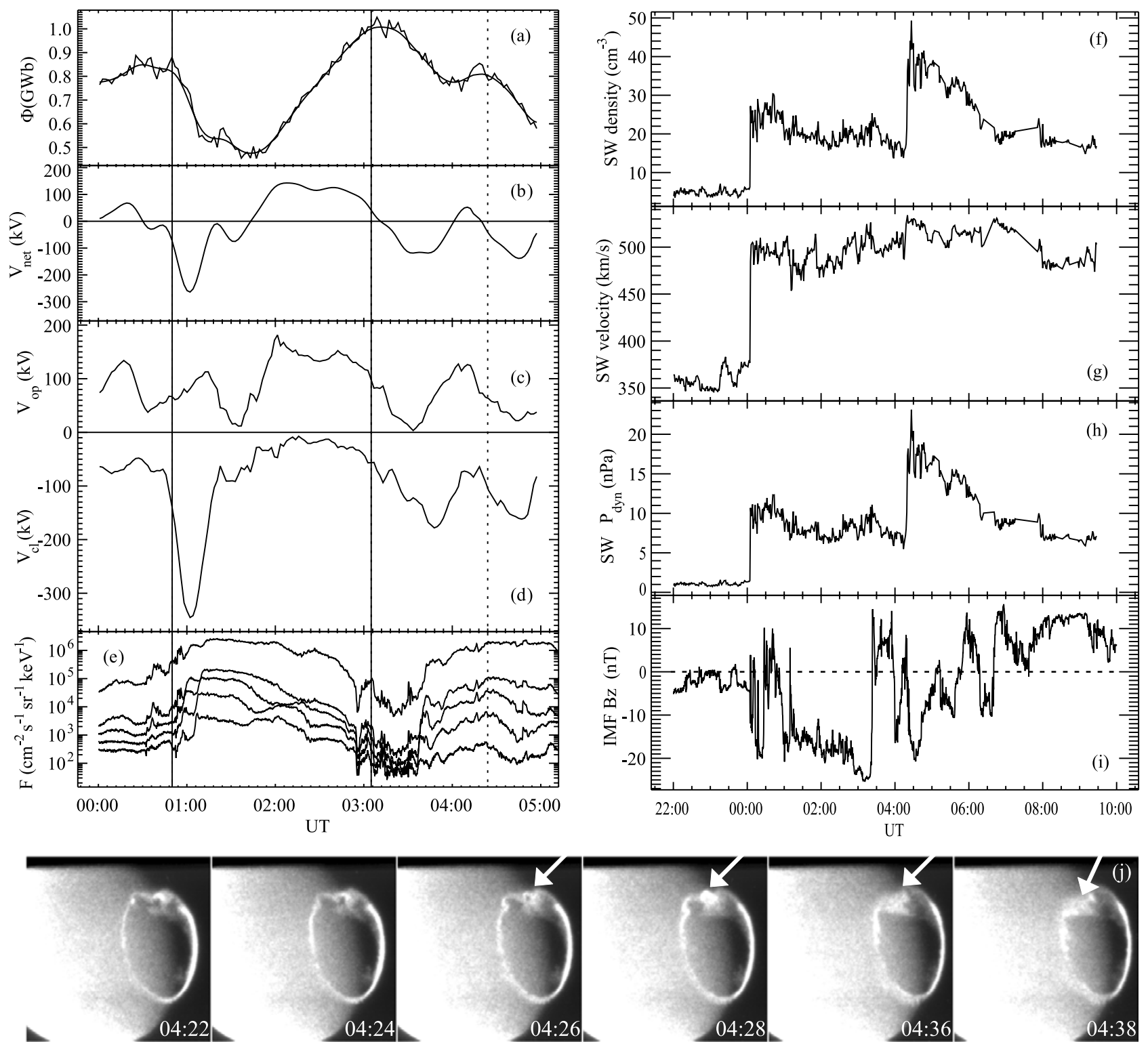

Figure 1. (a) Open magnetic flux, (b) net reconnection voltage, (c) opening and (d) closure voltages, and (e) energetic electron flux for the sawtooth event of 18 April 2001, and corresponding solar wind properties from the ACE satellite: (f) density, (g) velocity, (h) dynamic pressure, and (i) IMF $B_{z}$ component. The rapid variations in the open flux represent the noise level of the determination, such that smoothing is applied to produce the filtered curve. The solid vertical lines indicate two onsets reported by Huang et al. [2004]. The dotted vertical line indicates a maximum of the geosynchronous particle flux around 0424 UT. (j) The WIC images show the associated revival of the auroral activity that propagates eastward towards the noon sector, indicated by the overlaid arrows. The solar wind dynamic pressure front recorded by ACE shortly after 0000 UT was due to a sharp increase of both the solar wind density and velocity. It reached the Earth at 0048 UT and triggered a massive flux closure reaching $\sim-350 \mathrm{kV}$ that resulted in a reduction of the open flux by $\sim 0.3 \mathrm{GWb}$. The IMF was southward for most of the time, and the IMF Bz component reached $-26 \mathrm{nT}$.

with a sudden rise of both the solar wind speed from $\sim 370$ to $\sim 500 \mathrm{~km} / \mathrm{s}$ and of the density from $\sim 8$ to $\sim 26 \mathrm{~cm}^{-3}$ (Figures 1f and 1g), actually reached the Earth's magnetosphere shortly before 0048 UT and produced a dayside subauroral proton flash (DSPF) in the SI12 image at 0047:52 UT. A DSPF is known to be a typical signature of the compression of the dayside magnetosphere by a solar wind dynamic pressure front [Hubert et al., 2003], and further compression of the magnetotail is also known to be capable of stimulating the formation of an $\mathrm{X}$ line in the plasma sheet, where open magnetic flux is closed [Boudouridis et al., 2005; Hubert et al., 2006b].

[5] The closure voltage estimated shortly after the shock arrival reaches $\sim-350 \mathrm{kV}$ (Figure 1d), which is an extremely large value. Since most of this voltage comes from the motion of the polar boundary, the poor SuperDARN coverage at that time is not a key issue. By comparison, Hubert et al. [2006a] reported substorms for which the closure voltage reached $\sim-120$ to $-150 \mathrm{kV}$. The opening voltage that we estimate reaches an extreme value 
of $\sim 180 \mathrm{kV}$ at $\sim 0200 \mathrm{UT}$ (Figure 1c). This is due to the generally strong southward IMF and large solar wind velocity (Figures 1f and 1i) that favour reconnection on the dayside. Huang et al. [2004] report two other onsets at 0306 UT and 0600 UT. In addition, the energetic particle flux also slowly reaches a maximum around 0424 UT marked by the vertical dashed line (Figure 1e), which is not identified as one of the substorm onsets of the sawtooth sequence. The closure voltage that we compute progressively intensifies around $0300 \mathrm{UT}$, and reaches values as large as $-100 \mathrm{kV}$ around $0310 \mathrm{UT}$ (Figure 1d), i.e., typical of what can be expected at the time of a substorm expansion. Note that, around 0300 UT, the SuperDARN coverage was very good in the midnight sector. Around 0424 UT, the intensification of the closure voltage is conspicuous (Figure 1d), considering that the resolution of our method of estimation of the reconnection voltages is $\sim 12-14$ minutes. For this sawtooth event, the development of the sawtooth sequence in the measured particle flux appears to be intimately related with the process of magnetic reconnection during an interval in which the magnetosphere is strongly loaded with open magnetic flux.

[6] The FUV observations from the WIC and SI13 instruments also support the results presented above. The shockinduced flux closure starting at 0048 UT, which initiates the sawtooth sequence, has the typical auroral signatures of a shock-induced aurora in the WIC and SI13 images (not shown) [Meurant et al., 2003, 2004]: the brightening first occurs on the dayside oval, then brightenings reminiscent of a (multiple) expansion onset appear on the nightside, and the auroral intensification propagates to the entire oval. This takes place along with a massive retraction of the polar cap, as a consequence of the flux closure in the tail, consistently with the voltages of Figures $1 \mathrm{~b}$ and $1 \mathrm{~d}$. The SuperDARN radar recorded a strong enhancement of the ionospheric convection on the dayside right after the solar wind shock reached the magnetosphere, a phenomenon already reported by Boudouridis et al. [2007], until 0200 UT, i.e., at the end of the shock-induced flux closure episode. The oval remains active at later UT, and the polar cap size increases as a natural consequence of the strongly southward IMF and high opening voltage that we described above, consistently with the voltages of Figures $1 \mathrm{~b}$ and $1 \mathrm{c}$. An auroral brightening typical of a pseudobreakup is seen at $0256 \mathrm{UT}$, shortly followed by an expansion onset at 0305 UT. Pseudobreakups are known to close magnetic flux [Hubert et al., 2006a], but as the pseudobreakup and the onset are separated in time by 9 min only, our method cannot resolve both signatures (Figure 1d). This explains why the closure intensification seems to be progressive. In fact, a substorm expansion takes place while the auroral and magnetospheric activity remains high, powered by a strong production of open flux on the dayside that reaches the tail some time later, after the field lines have been dragged tailward by the solar wind flow. This constantly feeds the system with fresh open flux and energy that keep on powering the reconnection process that eventually lights up the aurora in the ionosphere. The auroral activity remains very high throughout the rest of the interval, during which it is very difficult to identify phases of the substorm cycle. There is however a signature seen in the FUV images which occurs in the dawn sector of the oval at 0426 UT and appears to propagate towards the dayside of the oval, which results in an obvious reduction of the polar cap area (Figure 1j). It is natural to think that this revival of activity is related to the flux closure found around 0424 UT (Figures 1a, 1b, and 1d), but one can hardly analyze it in terms of an isolated expansion phase. It rather appears as a reactivation of the expansion phase that started at 0305 UT. This reactivation is, again, powered by the large rate of flux opening on the dayside.

[7] The sawtooth interval presented above is thus characterized by (1) a very high rate of flux opening on the dayside that results in a magnetosphere strongly loaded with open flux, (2) a shock-induced flux closure with typical auroral signatures and an enormous rate of flux closure reaching $-350 \mathrm{kV}$, followed by (3) a typical substorm expansion phase starting with a substorm onset preceded by a pseudobreakup and (4) a reactivation of the activity associated with the latter expansion phase before the magnetosphere had a chance to return to a quiet state, powered by the ongoing production of open flux on the dayside.

\section{Sawtooth Event of 24 October 2002}

[8] Huang et al. [2004] also report a sawtooth event occurring between 0200 and 2400 UT on 24 October 2002. SI12 observations are available for that day between 1120 and $1610 \mathrm{UT}$, allowing us to estimate the location of the open/closed field line boundary during that interval. At that time, the SuperDARN radar network only offered a coverage of the dayside ionosphere, but the method developed by Ruohoniemi and Baker [1998] is used to reconstruct the ionospheric electric field across the whole polar region. Figures $2 \mathrm{a}, 2 \mathrm{c}$, and $2 \mathrm{~d}$ show the open magnetic flux and opening and closure voltages that we obtain for this interval. Based on particle measurements from the LANL 1991-080 spacecraft, Huang et al. [2004] report onsets at 1048, 1400 and $1650 \mathrm{UT}$. The IMAGE-FUV viewing geometry was too poor around 1048 UT to estimate the polar cap boundary location. The intensification of the particle flux around 1130 UT (Figure 2e) is also seen as an intensification of the FUV brightness and appears as a revival of the ongoing expansion phase (Figure 2j). We have useful data for the onset at 1400 UT only. At that time, the magnetosphere is loaded with $\sim 0.9 \mathrm{GWb}$ of open flux (Figure 2a). Throughout the interval, the amount of open flux remains high, with a lower bound of $\sim 0.5 \mathrm{GWb}$ and an upper bound of $\sim 0.9 \mathrm{Gwb}$. This is due to the orientation of the IMF which is southward throughout the interval, with a few northward excursions (Figure 2i). The solar wind dynamic pressure remains rather steady, although a $\sim 1 \mathrm{nPa}$ variation was recorded by the ACE satellite around 1100 UT (Figure 2h). The onset recorded at 1400 UT in the geosynchronous particle flux is clearly associated with an intensification of the closure voltage (Figure 2d). However, another intensification of the closure voltage is also recorded earlier, around $1225 \mathrm{UT}$, without a specific signature in the particle data, except that the magnitude of the flux slowly reaches a maximum value around that time (Figure 2e), in association with the evolution of the substorm expansion of the sawtooth sequence observed after 1048 UT. Note that, in our data, intensifications of the closure voltage are not always associated with a sharp signature in the measured particle flux. This may indicate that flux closure does not necessarily 

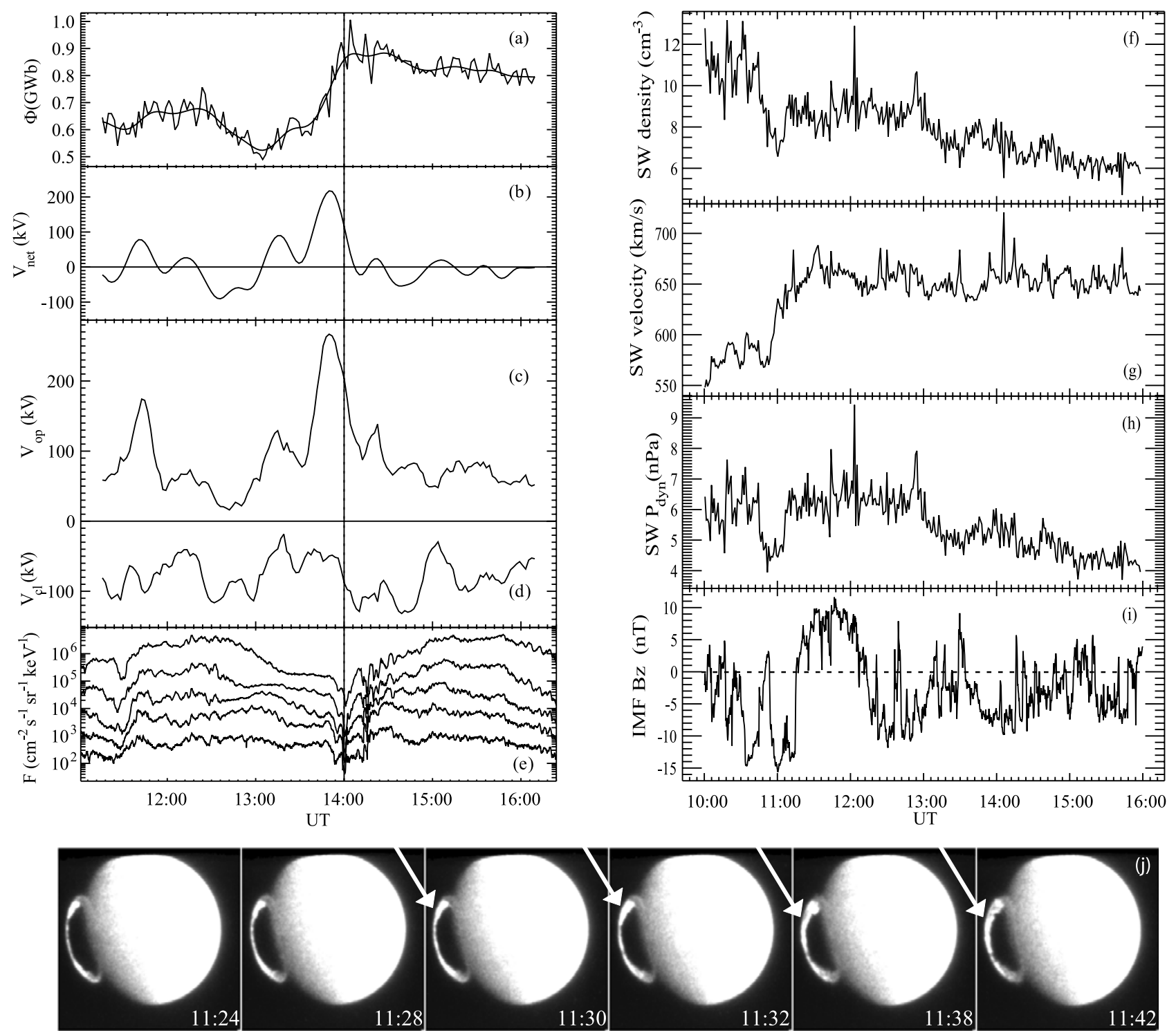

Figure 2. (a) Open magnetic flux, (b) net reconnection voltage, (c) opening and (d) closure voltages, and (e) energetic electron flux from the LANL 1991-080 spacecraft for the sawtooth event of 24 October 2002 and corresponding solar wind properties from the ACE satellite: (f) density, (g) velocity, (h) dynamic pressure, and (i) IMF $\mathrm{B}_{\mathrm{z}}$ component. The solid vertical line indicates the onset of the sawtooth sequence reported by Huang et al. [2004] at 1400 UT. (j) The WIC images show the revival of auroral activity that starts at $\sim 1130$ UT and propagates eastward, indicated by the overlaid arrows.

produce an additional particle injection, or that the injection is not strong enough to be identified, or that it can be localized so that it does not always appear in in-situ geosynchronous measurements.

[9] An expansion onset is seen at 1354 UT with the WIC camera. This onset evolves into an expansion phase in terms of auroral morphology, but the polar cap does not dramatically shrink, as can be expected from the evolution of the open magnetic flux (Figure 2a): the open magnetic flux does not dramatically decrease, as a consequence of the massive flux opening that takes place on the dayside, with the reconnection voltage reaching $\sim 270 \mathrm{kV}$ during the growth phase of the substorm (Figures $2 b$ and $2 c$ ), i.e., an extremely large value. One could argue that, as the SuperDARN coverage is not very good on the nightside at that time, this value could be overestimated. However, the net voltage, which does not depend on the SuperDARN data, peaks at $\sim 200 \mathrm{kV}$ at that time, so that the opening voltage undoubtedly reached a value larger than $200 \mathrm{kV}$. After the onset, the dayside flux opening voltage remains high (Figure 2c), so that the open flux value does not dramatically decrease despite the vigorous closure in the tail (Figures 2a, 2b, and $2 d)$. The closure of flux that we find around 1224 UT with a peak closure voltage at 1230 UT does not seem to have the typical auroral signatures of an expansion phase: no bright onset signature, no typical modification of the auroral morphology (bulge etc.). Nevertheless, it reduces the open flux of the magnetosphere over an interval of more than 30 minutes (Figures $2 \mathrm{a}, 2 \mathrm{~b}$, and $2 \mathrm{~d}$ ). The viewing geometry of the FUV instruments is not very favourable at that time, and details of the nightside aurora are difficult to analyze, but it is clear that the aurora remained bright throughout the 
interval. It is thus certainly possible that the intensification of the closure voltage that we record around 1225 UT could be a reactivation of the closure process associated with the expansion of the sawtooth sequence that was reported to start at 1048 UT by Huang et al. [2004]. In the absence of FUV data at that time, it is however hard to check the validity of this hypothesis: we have no measurement of the open flux and reconnection voltages at the time of the onset nor later, until the viewing geometry of the FUV instruments improves.

[10] It must be pointed out here that the main common characteristic of both events presented so far is the large value of the open magnetic flux due to a large rate of reconnection that opens flux on the dayside when the strongly southward IMF merges with the magnetospheric field. From that standpoint, although it does not appear in our data, it is also natural to think that when the IMF returns to a northward orientation or to a smaller southward component, the magnetosphere then also progressively returns to a more quiet state after it has closed the previously open flux, which ends the sawtooth sequence.

\section{Other Sawtooth Events}

[11] Other FUV observations coincident with sawtooth events reported by Huang et al. [2004] and Henderson et al. [2006] can be identified. We considered the sawtooth events that occurred on 11 August 2000, 4 October 2000, 14 October 2000, 22 October 2001, and 18 April 2002. Our results were of slightly lower quality for these intervals due to incomplete coverage of the proton aurora by the SI12 field of view, lower brightness of the proton aurora, higher background in the SI12 images due to different orientation of the instrument with respect to the Sun direction etc., during at least a part of the sawtooth interval with FUV coverage. It follows that the open flux that we compute is more noisy, as a result of the less accurate determination of the polar cap boundary. The reconnection voltages are then less accurate as well. Nevertheless, the same main tendency is found for these intervals: the sawtooth events are found to develop when the open magnetic flux is high. It was more than $1 \mathrm{GWb}$ during a large part of these intervals, and reached $\sim 1.2 \mathrm{GWb}$ during all of them. The high magnetic flux values result from the large reconnection voltage on the dayside during prolonged intervals, in agreement with the solar wind data from the ACE satellite showing a strongly southward IMF for most of the time during these intervals, which is consistent with a very efficient loading of the magnetosphere with open flux.

\section{Summary and Conclusions}

[12] The general picture that emerges from the case studies presented here is that a sawtooth event is actually a sequence of substorms taking place during an interval of strongly southward IMF that loads the magnetosphere with open flux, and keeps the amount of open flux at a high level despite the closure in the tail. As reported by Henderson et al. [2006], the closure process can be triggered by a solar wind shock, because an increase of the solar wind dynamic pressure favours the formation of an X line in the plasma sheet, but it is not a necessary condition. Further substorm expansions develop and relax the loaded magnetosphere, although there may be insufficient time for a complete recovery to take place before the closure rate intensifies again, as fresh open flux reaches the tail after vigorous opening on the dayside. The relation that we find between flux closure and the sawtooth events are consistent with that of Henderson et al. [2006], who reported a dipolarization of the Earth's magnetic field at geosynchronous orbit and an onset signature in the WIC images followed by a substorm expansion on 18 April 2002. The picture proposed here is consistent with the reconnection voltages that we compute and with the general characteristics of the auroral morphology. Ultimately, the factor that appears to be responsible for the sawtooth is a strongly southward IMF that overfeeds the magnetospheric system with open flux and energy during a prolonged time interval. We note that the strongly southward IMF condition was already reported previously [Henderson et al., 2006, and references therein]. It follows that the open flux produced is closed by reconnection in the tail to balance the production of open flux on a long time scale, which produces the sawtooth sequence of flux closure and geosynchronous particle flux activation on a shorter time scale.

[13] Acknowledgments. Jean-Claude Gérard and Benõcirc;t Hubert are supported by the Belgian Fund for Scientific Research (FRS). This work was funded by the PRODEX program of the European Space Agency (ESA). Work at Leicester was supported by STFC grant PP/D002117/1. ACE level 2 data were provided by N. F. Ness (MFI) and D. J. McComas (SWEPAM), and the ACE Science Centre. GOES-8 data were obtained thanks to CDAWeb. The LANL particle data were obtained from the LANL Geosynchronous Energetic Particle Data web page.

\section{References}

Akasofu, S.-I. (1964), The development of the auroral substorm, Planet. Space Sci., 12, 273.

Belian, R. D., T. E. Clayton, and G. D. Reeves (1995), Quasi-periodic, substorm-associated, global flux variations observed at geosynchronous orbit, in Space Plasmas: Coupling Between Small and Medium Scale Processes, Geophys. Monogr. Ser., vol. 86, edited by M. Ashour-Abdallah, T. Chang, and P. Dusenbery, p. 143, AGU, Washington, D. C.

Boudouridis, A., E. Zesta, L. R. Lyons, P. C. Anderson, and D. Lummerzheim (2005), Enhanced solar wind geoeffectiveness after a sudden increase in dynamic pressure during southward IMF orientation, J. Geophys. Res., 110, A05214, doi:10.1029/2004JA010704.

Boudouridis, A., L. R. Lyons, E. Zesta, and J. M. Ruohoniemi (2007), Dayside reconnection enhancement resulting from a solar wind dynamic pressure increase, J. Geophys. Res., 112, A06201, doi:10.1029/ 2006JA012141.

Cowley, S. W. H., and M. Lockwood (1992), Excitation and decay of solar wind-driven flows in the magnetosphere-ionosphere system, Ann. Geophys., 10, 103.

Dungey, J. W. (1961), Interplanetary field and the auroral zones, Phys. Res. Lett., $6,47$.

Henderson, M. G., G. D. Reeves, R. Skoug, M. F. Thomsen, M. H. Denton, S. B. Mende, T. J. Immel, P. C. Brandt, and H. J. Singer (2006), Magnetospheric and auroral activity during the 18 April 2002 sawtooth event, J. Geophys. Res., 111, A01S90, doi:10.1029/2005JA011111.

Huang, C. S., G. Le, and G. D. Reeves (2004), Periodic magnetospheric substorms during fluctuating interplanetary magnetic $B_{z}$, Geophys. Res. Lett., 31, L14801, doi:10.1029/2004GL020180.

Hubert, B., J.-C. Gérard, S. A. Fuselier, and S. B. Mende (2003), Observation of dayside subauroral proton flashes with the IMAGE-FUV imagers, Geophys. Res. Lett., 30(3), 1145, doi:10.1029/2002GL016464.

Hubert, B., S. E. Milan, A. Grocott, C. Blockx, S. W. H. Cowley, and J.-C. Gérard (2006a), Dayside and nightside reconnection rates inferred from IMAGE FUV and Super Dual Auroral Radar Network data, J. Geophys. Res., 111, A03217, doi:10.1029/2005JA011140.

Hubert, B., M. Palmroth, T. V. Laitinen, P. Janhunen, S. E. Milan, A. Grocott, S. W. H. Cowley, T. Pulkkinen, and J.-C. Gérard (2006b), Compression of the Earth's magnetotail by interplanetary shocks directly drives transient magnetic flux closure, Geophys. Res. Lett., 33, L10105, doi:10.1029/2006GL026008. 
McPherron, R. L. (1970), Growth phase of magnetospheric substorms, J. Geophys. Res., 75, 5592

Mende, S. B., et al. (2000a), Far ultraviolet imaging from the IMAGE spacecraft: 1. System design, Space Sci. Rev., 91, 243.

Mende, S. B., et al. (2000b), Far ultraviolet imaging from the IMAGE spacecraft: 3. Spectral imaging of Lyman alpha and OI $135.6 \mathrm{~nm}$, Space Sci. Rev., 91, 287.

Meurant, M., J.-C. Gérard, B. Hubert, V. Coumans, C. Blockx, N. Østgaard, and S. B. Mende (2003), Dynamics of global scale electron and proton precipitation induced by a solar wind pressure pulse, Geophys. Res. Lett. 30(20), 2032, doi:10.1029/2003GL018017.

Meurant, M., J.-C. Gérard, C. Blockx, B. Hubert, and V. Coumans (2004), Propagation of electron and proton shock-induced aurora and the role of the interplanetary magnetic field and solar wind, J. Geophys. Res., 109, A10210, doi:10.1029/2004JA010453.

Milan, S. E., G. Provan, and B. Hubert (2007), Magnetic flux transport in the Dungey cycle: A survey of dayside and nightside reconnection rates, J. Geophys. Res., 112, A01209, doi:10.1029/2006JA011642.

Ruohoniemi, J. M., and K. B. Baker (1998), Large scale imaging of high latitude convection with Super Dual Auroral Radar Network HF radar observations, J. Geophys. Res., 103, 20,797.

S. W. H. Cowley, A. Grocott, and S. E. Milan, Department of Physics and Astronomy, University of Leicester, Leicester LE1 7RH, UK.

J. C. Gérard and B. Hubert, Laboratory for Planetary and Atmospheric Physics, University of Liège, B-4000 Liège, Belgium. (b.hubert@ulg.ac.be) 